\title{
Mirror versus naive assignment in chiral models for the nucleon
}

\author{
Susanna Gallas and Francesco Giacosa \\ Institut für Theoretische Physik, Johann Wolfgang Goethe - Universität \\ Max von Laue-Str. 1, 60438 Frankfurt am Main, Germany
}

\begin{abstract}
In the framework of chiral model(s) based on the linear realization of chiral symmetry, we investigate the low-energy phenomenological properties of the nucleon and its chiral partner (identified with either $N(1535)$ or $N(1650)$ ) in the naive assignment and compare the results with the ones obtained in the mirror assignment. We find that, within the naive assignment, we are not able to reproduce the experimental value of the isospin-odd scattering length, while the mirror assignment is in good agreement with it for both choices $N(1535)$ and $N(1650)$. The isospin-even scattering length is not yet conclusive in either assignment because it depends crucially on the poorly known scalar mesonic sector. The decay with $\Gamma_{N(1535) \rightarrow N \eta}$ turns out to be far too small in both the naive and mirror assignments, while $\Gamma_{N(1650) \rightarrow N \eta}$ is described correctly by both of them. In conclusions, the mirror assignment with $N(1650)$ as the chiral partner of the nucleon is the favored configuration.
\end{abstract}

\section{Introduction}

The question of the mass generation of particles is crucial in modern high energy physics. The standard model predicts the existence of a scalar boson, the Higgs particle, which is capable of giving mass to the fundamental particles by the spontaneous breaking of the electroweak symmetry. Now, what about the mass of composite objects like the baryons, most notably the nucleon? The sum of the mass of the three quarks that form a nucleon is not equal to the mass of the baryon, but is much smaller $(<5 \%)$. Where does the dominant contribution to the nucleon mass come from?

In this work we want to shed some light on this question. We focus on two mechanisms in which we can introduce the nucleon and its chiral partner, denoted as $N$ and $N^{*}$ respectively, by testing the consequences in each case. The field $N^{*}$ is usually identified with the resonance $N(1535)$, but $N(1650)$ is also a viable candidate [1. We study both scenarios in this work.

One way to introduce the chiral partner of the nucleon is via the naive assignment [2, 3, 4,. In this assignment the mass of the nucleon (and that of its chiral partner) is generated only through spontaneous chiral symmetry breaking, since a mass term would not be chirally invariant. (We neglect here the small contribution of the nonzero bare quark masses.)

The introduction of an explicit mass term, which should occur in a chirally invariant way, is only possible within the mirror assignment. The mirror assignment was analyzed for the first time in Ref. [2] and there dismissed as unusable, as it led to the unphysical conclusion that the coupling between nucleons and pions vanishes. This problem was solved later by introducing all terms allowed by chiral symmetry. The interest in the mirror assignment has been revitalized by the work in Ref. [5], after which it has been investigated in a variety of works studying the phenomenology of baryons in the vacuum and at nonzero density [6, 7, 8, 9, 10, 11, 12, 13, 14.

It is still an open question which is the correct assignment in nature. To this end, both assignments should be investigated and validated by experimental data such as the axial coupling constants, the decays $N^{*} \rightarrow N \pi$ and $N^{*} \rightarrow N \eta$, and the $N \pi$ scattering lengths: this is the aim of the present 
work. Namely, while in our previous study of Ref. 8 w we concentrated, in the framework of the socalled extended Linear Sigma Model (eLSM), on the mirror assignment only, here we construct for the first time the eLSM version also for the naive assignment. The eLSM contains, besides the usual (pseudo)scalar mesons, also (axial-)vector degrees of freedom; their presence changes substantially the properties of baryon phenomenology both in the naive and mirror assignments allowing for a correct description of the axial coupling constants of $N$ and $N^{*}$. Moreover, in the naive assignment, the absence of (axial-)vector states implies a complete decoupling of the fields $N$ and $N^{*}[6$, thus also implying the (unphysical) vanishing of the decay $N^{*} \rightarrow N \pi$. The introduction of (axial-)vector d.o.f. implies a residual $N^{*} N \pi$ coupling, thus making also the naive assignment realistic. However, we shall find that, even in this more complete treatment, the naive assignment is not capable to reproduce the isospin-odd pion-nucleon scattering length. We thus arrive at the conclusion that the mirror assignment is favoured to be the correct way to incorporate baryons in (linear) chiral models.

It should be stressed that our approach is not designed to reach the accuracy that chiral perturbation theory delivers in the description of pion-nucleon scattering. Namely, the latter approach is tailormade to study the interaction of nucleons with the quasi-Goldstone bosons of low-energy QCD, the pions, in a systematic framework, see e.g. Refs. [15, 16] and refs. therein. However, our linear chiral approach has the advantage to incorporate from the very beginning massive resonances (i.e., the chiral partners), and is thus suited to study their decays, as shown in Ref. 8 8 in the baryonic sector and in Refs. [17, 18 in the mesonic sector. As a consequence of the chiral invariance of the Lagrangian, the low-energy theorems are fulfilled. Moreover, our approach can be easily implemented at nonzero temperature and density to investigate the chiral phase transition within hadronic matter [10, 19]. In this context the use of the correct assignment is mandatory, because it strongly affects the behavior of physical quantities in a dense and hot medium.

This paper is organized as follows: in section 2 the Lagrangians of both assignments are studied, in section 3 the results are shown and finally in section 4 conclusions and outlooks are presented.

Our units are $\hbar=c=1$, the metric tensor is $g^{\mu \nu}=\operatorname{diag}(+,-,-,-)$.

\section{Mirror and naive assignments}

In this section we present the baryonic part of the eLSM in the mirror and naive assignments, respectively. We concentrate to the two-flavour case, $N_{f}=2$. The scalar and pseudoscalar fields are included in the matrix

$$
\Phi=\sum_{a=0}^{3} \phi_{a} t_{a}=\left(\sigma+i \eta_{N}\right) t^{0}+\left(\vec{a}_{0}+i \vec{\pi}\right) \cdot \vec{t},
$$

where $\vec{t}=\vec{\tau} / 2$, with the vector of Pauli matrices $\vec{\tau}$, and $t^{0}=\mathbf{1}_{2} / 2$. Under $U\left(N_{f}=2\right)_{R} \times U\left(N_{f}=2\right)_{L}$ chiral symmetry, $\Phi$ transforms as $\Phi \rightarrow U_{L} \Phi U_{R}^{\dagger}$. The vector and axial-vector fields are contained in the matrices

$$
\begin{aligned}
V^{\mu} & =\sum_{a=0}^{3} V_{a}^{\mu} t_{a}=\omega^{\mu} t^{0}+\vec{\rho}^{\mu} \cdot \vec{t} \\
A^{\mu} & =\sum_{a=0}^{3} A_{a}^{\mu} t_{a}=f_{1}^{\mu} t^{0}+{\overrightarrow{a_{1}}}^{\mu} \cdot \vec{t} .
\end{aligned}
$$

From these fields, we define the right- and left-handed vector fields as $R^{\mu} \equiv V^{\mu}-A^{\mu}$ and $L^{\mu} \equiv V^{\mu}+A^{\mu}$. Under $U(2)_{R} \times U(2)_{L}$ these fields change as $R^{\mu} \rightarrow U_{R} R^{\mu} U_{R}^{\dagger}, L^{\mu} \rightarrow U_{L} L^{\mu} U_{L}^{\dagger}$. The identification of mesons with particles listed in Ref. [1] is as follows: the fields $\vec{\pi}$ and $\eta_{N}$ correspond to the pion and the $S U(2)$ counterpart of the $\eta$ meson, $\eta_{N} \equiv(\bar{u} u+\bar{d} d) / \sqrt{2}$, with a mass of about $700 \mathrm{MeV}$. This value can be obtained by unmixing the physical $\eta$ and $\eta^{\prime}$ mesons, which also contain $\bar{s} s$ contributions. The fields $\omega^{\mu}$ and $\vec{\rho}^{\mu}$ represent the vector mesons $\omega(782)$ and $\rho(770)$, and the fields $f_{1}^{\mu}$ and ${\overrightarrow{a_{1}}}^{\mu}$ represent the axial-vector mesons $f_{1}(1285)$ and $a_{1}(1260)$, respectively. 
The identification of the $\sigma$ and $\vec{a}_{0}$ fields is controversial, the possible pairs are $\left\{f_{0}(500), a_{0}(980)\right\}$ and $\left\{f_{0}(1370), a_{0}(1450)\right\}$, the latter one being in agreement with the phenomenology, see Refs. [17, 18, in which the mesonic part of the Lagrangian is presented in depth. Some conclusions are however essential for the following studies of the baryonic part as well: the chiral condensate $\sigma_{0}=\langle 0|\sigma| 0\rangle=Z f_{\pi}$ emerges upon spontaneous chiral symmetry breaking in the mesonic sector. The parameter $f_{\pi}=92.2 \mathrm{MeV}$ is the pion decay constant and $Z=1.81$ is the wave-function renormalization constant of the pseudoscalar fields (see the determination of $Z$ in Sec. 3).

Turning to the baryon sector, we have the baryon doublets $\Psi_{1}$ and $\Psi_{2}$, where $\Psi_{1}$ has positive parity and $\Psi_{2}$ negative parity. The physical fields $N$ and $N^{*}$ are related to the spinors $\Psi_{1}$ and $\Psi_{2}$ through:

$$
\begin{aligned}
& \Psi_{1}=\frac{1}{\sqrt{2 \cosh \delta}}\left(N e^{\delta / 2}+\gamma_{5} N^{*} e^{-\delta / 2}\right), \\
& \Psi_{2}=\frac{1}{\sqrt{2 \cosh \delta}}\left(\gamma_{5} N e^{-\delta / 2}-N^{*} e^{\delta / 2}\right) .
\end{aligned}
$$

The quantity $\delta$ parametrizes the mixing between $\Psi_{1}$ and $\Psi_{2}$ : for $\delta \rightarrow \infty$ the mixing of the fields disappears, implying $\Psi_{1}=N$ and $\Psi_{2}=N^{*}$; for $\delta=0$ the mixing is maximal.

The fundamental difference between the mirror assignment and the naive assignment is the transformation of these baryon doublets under the chiral group $U(2)_{R} \times U(2)_{L}$, which we discuss in the following.

\section{Mirror assignment}

The nucleons $N$ and $N^{*}$ belong to the same multiplet and thus are true chiral partners 6 , 20]. In this assignment the left-handed and the right-handed parts of $\Psi_{1}$ and $\Psi_{2}\left(\Psi_{i}=\Psi_{i, R}+\Psi_{i, L}\right.$, $i=1,2)$ transform as:

$$
\begin{aligned}
& \Psi_{1 R} \rightarrow U_{R} \Psi_{1 R}, \Psi_{1 L} \rightarrow U_{L} \Psi_{1 L}, \\
& \Psi_{2 R} \rightarrow U_{L} \Psi_{2 R}, \Psi_{2 L} \rightarrow U_{R} \Psi_{2 L},
\end{aligned}
$$

i.e. $\Psi_{2}$ transforms in a "mirror way" under chiral transformation. These field transformations allow to write down a baryonic Lagrangian with a chirally invariant mass term for the fermions, which is parametrized by $m_{0}$. The full Lagrangian of the eLSM (for $N_{f}=2$ ) in the baryonic sector reads [8]:

$$
\begin{aligned}
\mathcal{L}_{\text {mirror }} & =\bar{\Psi}_{1 L} i \gamma_{\mu} D_{1 L}^{\mu} \Psi_{1 L}+\bar{\Psi}_{1 R} i \gamma_{\mu} D_{1 R}^{\mu} \Psi_{1 R}+\bar{\Psi}_{2 L} i \gamma_{\mu} D_{2 R}^{\mu} \Psi_{2 L}+\bar{\Psi}_{2 R} i \gamma_{\mu} D_{2 L}^{\mu} \Psi_{2 R} \\
& -\widehat{g}_{1}\left(\bar{\Psi}_{1 L} \Phi \Psi_{1 R}+\bar{\Psi}_{1 R} \Phi^{\dagger} \Psi_{1 L}\right)-\widehat{g}_{2}\left(\bar{\Psi}_{2 L} \Phi^{\dagger} \Psi_{2 R}+\bar{\Psi}_{2 R} \Phi \Psi_{2 L}\right) \\
& -m_{0}\left(\bar{\Psi}_{1 L} \Psi_{2 R}-\bar{\Psi}_{1 R} \Psi_{2 L}-\bar{\Psi}_{2 L} \Psi_{1 R}+\bar{\Psi}_{2 R} \Psi_{1 L}\right)
\end{aligned}
$$

where $D_{1 R}^{\mu}=\partial^{\mu}-i c_{1} R^{\mu}, D_{1 L}^{\mu}=\partial^{\mu}-i c_{1} L^{\mu}$, and $D_{2 R}^{\mu}=\partial^{\mu}-i c_{2} R^{\mu}, D_{2 L}^{\mu}=\partial^{\mu}-i c_{2} L^{\mu}$ are the covariant derivatives for the nucleonic fields, with the dimensionless coupling constants $c_{1}$ and $c_{2}$. The interaction of the baryonic fields with the scalar and pseudoscalar mesons is parametrized by the dimensionless coupling constants $\widehat{g}_{1}$ and $\widehat{g}_{2}$. The last term in Eq. (5)

$$
\mathcal{L}_{m_{0}}=-m_{0}\left(\bar{\Psi}_{1 L} \Psi_{2 R}-\bar{\Psi}_{1 R} \Psi_{2 L}-\bar{\Psi}_{2 L} \Psi_{1 R}+\bar{\Psi}_{2 R} \Psi_{1 L}\right)
$$

is the chirally invariant mass term for the baryons. The masses of the nucleon and its chiral partner are obtained by diagonalizing the corresponding mass matrix in the Lagrangian:

$$
\begin{aligned}
& m_{N}=\sqrt{\left[\frac{\widehat{g}_{1}+\widehat{g}_{2}}{4}\right]^{2} \sigma_{0}^{2}+m_{0}^{2}}+\frac{\widehat{g}_{1}-\widehat{g}_{2}}{4} \sigma_{0}, \\
& m_{N^{*}}=\sqrt{\left[\frac{\widehat{g}_{1}+\widehat{g}_{2}}{4}\right]^{2} \sigma_{0}^{2}+m_{0}^{2}}-\frac{\widehat{g}_{1}-\widehat{g}_{2}}{4} \sigma_{0} .
\end{aligned}
$$


The parameter $\delta$ entering in Eqs. (3) reads:

$$
\sinh \delta=\frac{\left(\widehat{g}_{1}+\widehat{g}_{2}\right) \sigma_{0}}{4 m_{0}} .
$$

From these expressions it is evident that the baryonic masses are generated by chiral symmetry breaking trough the appearance of the chiral condensate $\sigma_{0}$ and by the mass parameter $m_{0}$. In particular, the chiral condensate originates a splitting of the masses in the chirally broken phase. In the limit $\sigma_{0} \rightarrow 0$ both masses become degenerate, but do not vanish: $m_{N}=m_{N^{*}}=m_{0} \neq 0$ (see Fig. 1, where the masses are plotted as function of $\sigma_{0}$ ). The numerical value of $m_{0}$ is determined through a fit procedure, see Sec. 3 and Ref. 8 for details. In the case $N^{*} \equiv$ $N(1535)$ one obtains $m_{0} \simeq 500 \mathrm{MeV}$, while for $N^{*} \equiv N(1650)$ one obtains a even larger value $m_{0} \simeq 700 \mathrm{MeV}$. These results mean that $m_{0}$ is sizable and is therefore an important component of the baryonic masses. These values are larger than the one originally found in the pioneering work of Ref. [5], in which $m_{0} \simeq 200 \mathrm{MeV}$ was found.

The expressions for the axial coupling constants of the nucleon and its chiral partner are given by $[8$ :

$$
g_{A}^{N}=\frac{1}{2 \cosh \delta}\left(g_{A}^{(1)} e^{\delta}+g_{A}^{(2)} e^{-\delta}\right), g_{A}^{N^{*}}=\frac{1}{2 \cosh \delta}\left(g_{A}^{(1)} e^{-\delta}+g_{A}^{(2)} e^{\delta}\right),
$$

where

$$
g_{A}^{(1)}=1-\frac{c_{1}}{g_{1}}\left(1-\frac{1}{Z^{2}}\right), g_{A}^{(2)}=-1+\frac{c_{2}}{g_{1}}\left(1-\frac{1}{Z^{2}}\right),
$$

where the parameter $g_{1}=6.1$ describes the interaction of (axial-)vector mesons with (pseudo)scalar mesons [17. It is evident that, due to the presence of the parameter $c_{1}$ and $c_{2}$ which arise from the interaction terms of the baryons with (axial-)vector degrees of freedom, the axial-coupling constant $g_{A}^{N}$ can be larger than unity. (The limitation $\left|g_{A}^{N}\right| \leq 1$ holds if vector mesons are not included [21]). This shows that the inclusion of the (axial-)vector states is important for the correct description of the phenomenology, see the results in the next section.

Note, the Lagrangian of Eq. (5) is invariant under chiral transformation. This means that in the present version of the model the explicit breaking of chiral symmetry is confined to the mesonic sector, in which the pions acquire a small but nonzero mass. Indeed, it would not be difficult to include an explicit symmetry breaking mass term also in the baryonic sector, but in view of the purposes of this work and in order to keep the number of parameters as small as possible, we leave this task for the future. In particular, such an explicit symmetry breaking will be unavoidable when the three-flavor version of the model will be studied. However, it is important to stress that even if no explicit symmetry breaking term in the baryonic Lagrangian is present the so-called pion-nucleon sigma term $\sigma_{\pi N}$ (see for instance Refs. 4, 22, 23, 24, and refs. therein), does not vanish [3. In fact, this term, which describes the contribution to the nucleon mass which arises from the explicit symmetry breaking, acquires a nonzero (and quite sizable) contribution from the explicit symmetry breaking encoded in the chiral condensate $\sigma_{0}$. The numerical results for $\sigma_{\pi N}$ are discussed in the next session.

2. Naive assignment

In this assignment both the left-handed and the right-handed part of the states $\Psi_{1}$ and $\Psi_{2}$ behave in the same way under chiral transformation:

$$
\begin{aligned}
& \Psi_{1 R} \rightarrow U_{R} \Psi_{1 R}, \Psi_{1 L} \rightarrow U_{L} \Psi_{1 L}, \\
& \Psi_{2 R} \rightarrow U_{R} \Psi_{2 R}, \Psi_{2 L} \rightarrow U_{L} \Psi_{2 L} .
\end{aligned}
$$




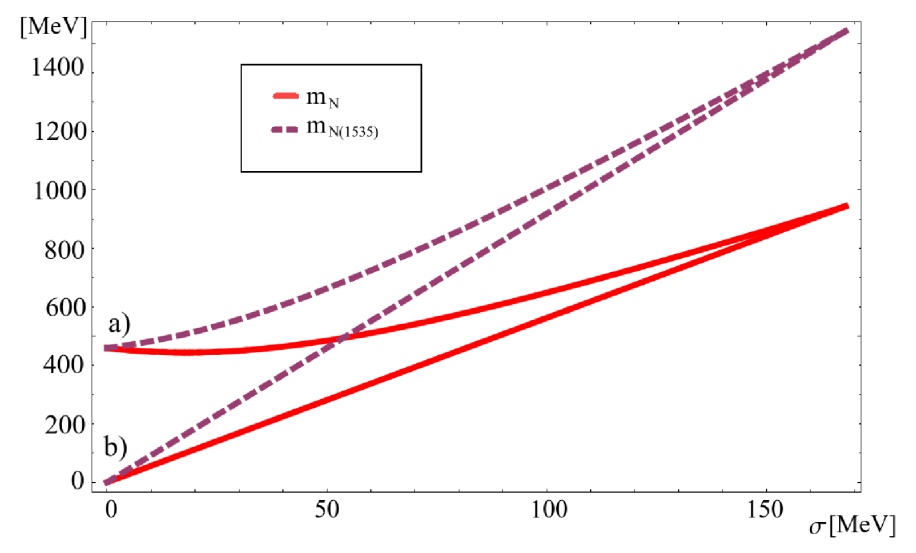

Figure 1: The masses of the baryons $N$ and $N^{*}$ in the a) mirror assignment (Eq. (7), parameters from Eq. (18)) and b) in the naive assignment (Eq. (15), parameters from Eq. (24)) as a function of the chiral condensate $\sigma_{0}$ : a) for vanishing $\sigma_{0}$ the masses acquire the same non-zero value $m_{N}=m_{N^{*}}=m_{0}$, while in b) they vanish in this limit. On the very right of the plot, the masses reach their physical values, $m_{N}=939 \mathrm{MeV}$ and $m_{N^{*}}=1535 \mathrm{MeV}$, in both assignments.

By introducing the chiral partner in this way, a nucleon mass term $\sim m_{N} \bar{\Psi} \Psi$ is not allowed because it explicitly breaks chiral symmetry. The Lagrangian in the naive assignment reads:

$$
\begin{aligned}
\mathcal{L}_{\text {naive }} & =\bar{\Psi}_{1 L} i \gamma_{\mu} D_{1 L}^{\mu} \Psi_{1 L}+\bar{\Psi}_{1 R} i \gamma_{\mu} D_{1 R}^{\mu} \Psi_{1 R}+\bar{\Psi}_{2 L} i \gamma_{\mu} D_{2 L}^{\mu} \Psi_{2 L}+\bar{\Psi}_{2 R} i \gamma_{\mu} D_{2 R}^{\mu} \Psi_{2 R} \\
& +c_{12} \bar{\Psi}_{1 R} \gamma_{\mu} R^{\mu} \Psi_{2 R}+c_{12} \bar{\Psi}_{2 R} \gamma_{\mu} R^{\mu} \Psi_{1 R}-c_{12} \bar{\Psi}_{1 L} \gamma_{\mu} L^{\mu} \Psi_{2 L}-c_{12} \bar{\Psi}_{2 L} \gamma_{\mu} L^{\mu} \Psi_{1 L} \\
& -\widehat{g}_{1}\left(\bar{\Psi}_{1 L} \Phi \Psi_{1 R}+\bar{\Psi}_{1 R} \Phi^{\dagger} \Psi_{1 L}\right)-\widehat{g}_{2}\left(\bar{\Psi}_{2 L} \Phi \Psi_{2 R}+\bar{\Psi}_{2 R} \Phi^{\dagger} \Psi_{2 L}\right) \\
& +\widehat{g}_{12} \bar{\Psi}_{1 R} \Phi^{\dagger} \Psi_{2 L}-\widehat{g}_{12} \bar{\Psi}_{1 L} \Phi \Psi_{2 R}+\widehat{g}_{12} \bar{\Psi}_{2 L} \Phi \Psi_{1 R}-\widehat{g}_{12} \bar{\Psi}_{2 R} \Phi^{\dagger} \Psi_{1 L} .
\end{aligned}
$$

Note that, indeed, some terms not permitted in the mirror assignment turn out to be chirally invariant in the naive case. They are parametrized by the coupling constants $c_{12}$ and $\widehat{g}_{12}$. The spinors $\Psi_{1}$ and $\Psi_{2}$ are replaced by the physical fields as dictated by eq. (3), where now the mixing parameter $\delta$ is given by:

$$
\sinh \delta=\frac{\widehat{g}_{1}+\widehat{g}_{2}}{2 \widehat{g}_{12}}
$$

There are however crucial differences between the term proportional to $\widehat{g}_{12}$ in Eq. (12) w.r.t. the term proportional to $m_{0}$ in the mirror case, Eq. (5). First, the parameter $\widehat{g}_{12}$ is dimensionless, while $m_{0}$ has dimension of energy. Second, the $\widehat{g}_{12}$-term involves the same interactions as the terms proportional to $\widehat{g}_{1}$ and $\widehat{g}_{2}$ and therefore can be completely eliminated by a suitable field transformation. (Such an operation is not possible in the mirror case, where the elimination of the mixing for the quadratic mass terms does not imply an elimination of the effects of the mixing in the interaction terms). Then, in the naive assignment, by expressing the Lagrangian in terms of the physical fields $N$ and $N^{*}$, we obtain the following form in which only terms which couple the nucleon, its chiral partner and the pion are included:

$$
\begin{aligned}
\mathcal{L}_{\text {naive }} & =\bar{N} i \gamma_{\mu} \partial^{\mu} N+\bar{N}^{*} i \gamma_{\mu} \partial^{\mu} N^{*}-\tilde{\widehat{g}}_{1} \bar{N}\left(\sigma+Z i \gamma_{5} \vec{\pi} \cdot \vec{\tau}\right) N-\tilde{\widehat{g}}_{2} \bar{N}^{*}\left(\sigma+Z i \gamma_{5} \vec{\pi} \cdot \vec{\tau}\right) N^{*} \\
& -Z w \tilde{c}_{1} \bar{N} \gamma_{5} \gamma^{\mu} \partial_{\mu} \vec{\pi} \cdot \vec{\tau} N-Z w \tilde{c}_{2} \bar{N}^{*} \gamma_{5} \gamma^{\mu} \partial_{\mu} \vec{\pi} \cdot \vec{\tau} N^{*} \\
& -Z w \tilde{c}_{12}\left(\bar{N} \gamma^{\mu} \partial_{\mu} \vec{\pi} \cdot \vec{\tau} N^{*}+\bar{N}^{*} \gamma^{\mu} \partial_{\mu} \vec{\pi} \cdot \vec{\tau} N\right)+\ldots
\end{aligned}
$$


The coupling constants with "tilde" are combinations of the original couplings, $\widehat{g}_{1}, \widehat{g}_{2}, \widehat{g}_{12}, c_{1}$, $c_{2}, c_{12}$ and $\delta$, reducing in this way the number of parameters from six to five (see Appendix B): $\tilde{\widehat{g}}_{1}, \tilde{\widehat{g}}_{2}, \tilde{c}_{1}, \tilde{c}_{2}$ and $\tilde{c}_{12}$. The masses of $N$ and $N^{*}$ can be easily obtained from Eq. (14) (see Fig. 1 for a plot as function of $\sigma_{0}$ ):

$$
m_{N}=\tilde{\widehat{g}}_{1} \sigma_{0} \quad \text { and } \quad m_{N^{*}}=\tilde{\widehat{g}}_{2} \sigma_{0} .
$$

In terms of $N$ and $N^{*}$ there are no interactions of the type $\bar{N} i \gamma_{5} \vec{\pi} \cdot \vec{\tau} N^{*}$. Therefore the coupling between the nucleon, its chiral partner and the pion takes the simple form $\bar{N}^{*} \gamma_{5} \gamma^{\mu} \partial_{\mu} \vec{\pi} \cdot \vec{\tau} N+$ h.c., which stems solely from the shift of the axial-vector meson $\vec{a}_{1} \rightarrow \vec{a}_{1}+Z w \partial_{\mu} \vec{\pi}$, which guarantees the disappearance of non-diagonal terms in the mesonic sector of the theory [17, 18, 25. Explicitly, the parameter $w$ reads:

$$
w=\frac{g_{1} \sigma_{0}}{m_{a_{1}}^{2}},
$$

where $m_{a_{1}}=1230 \mathrm{MeV}$ is the mass and the already introduced parameter $g_{1}=6.1$ describes the interaction of (axial-)vector mesons with (pseudo)scalar ones. As a consequence, the quantity $w$ takes the numerical value $w=67 \cdot 10^{-5} \mathrm{MeV}^{-1}$.

In a scenario in which (axial-)vector mesons decouple, the parameter $g_{1}$ vanishes, and so does the parameter $w$ in Eq. (16). Therefore the nucleon and its chiral partner decouple completely [6] and decay processes such as $N^{*} \rightarrow N \pi$ are not be possible [6]. Thus, the naive assignment without (axial-)vector mesons is explicitly unphysical and can be immediately discarded, because the decay rate $N^{*} \rightarrow N \pi$ has been measured to be nonzero for both candidates $N(1535)$ and $N(1650)$. With the inclusion of (axial-)vector d.o.f. this evident drawback is eliminated but, as we shall show, the isospin-odd scattering length turns out to be anyhow poorly described in the naive assignment.

The axial coupling constants of the nucleon and its chiral partner are given by:

$$
g_{A}^{(N)}=1-2 Z w f_{\pi} \tilde{c}_{1}, \quad g_{A}^{\left(N^{*}\right)}=1-2 Z w f_{\pi} \tilde{c}_{2},
$$

where $w$ is given by Eq. (16). It is also here visible that the axial coupling constant $g_{A}^{(N)}$ is not limited by unity due to the interaction with (axial-)vector states. Moreover, just as in the mirror case, the Lagrangian (12) does not include terms with explicit breaking of chiral symmetry, but the pion-nucleon sigma term $\sigma_{\pi N}$ does not vanish, see Sec. 3.2 for a numerical evaluation.

\section{Results}

\subsection{Mirror assignment}

We first review the case in which $N^{*}$ is identified with the resonance $N(1535)$. We perform a fit of the four parameters $m_{0}, c_{1}, c_{2}$, and $Z$ by using the following five experimental and lattice quantities:

(i) The axial-coupling constant of the nucleon: $g_{A}^{(N, \exp )}=1.267 \pm 0.004$ [1]. (ii) The lattice result for the axial-coupling constant of $N^{*}(1535): g_{A}^{\left(N^{*}\right)}=0.2 \pm 0.3$ [26]. (iii) The decay width of $N(1535)$ into $N \pi: \Gamma_{N^{*} \rightarrow N \pi}=67.5 \pm 11.2 \mathrm{MeV}$ [1]. (iv) The electromagnetic decay of the $a_{1}(1230)$ meson into $\pi \gamma: \Gamma_{a_{1} \rightarrow \gamma \pi}=(0.640 \pm 0.250) \mathrm{MeV}$ [1]. (v) The strong decay of the $a_{1}(1230)$ meson into $\rho \pi$ : $\Gamma_{a_{1} \rightarrow \rho \pi}=(425 \pm 175) \mathrm{MeV}$. This value is obtained by using the range 250-600 MeV quoted by [1], under the assumption that this decay channel is dominant. This is in well agreement with the detailed experimental analysis of Ref. 27] and with the recent phenomenological study of the nonet of axialvector mesons in Ref. [28]. The results read $\left(\chi_{\min }^{2}=0.64\right)$ :

$$
m_{0}=459 \pm 117 \mathrm{MeV}, c_{1}=-2.65 \pm 0.18, c_{2}=10.2 \pm 2.6, Z=1.81 \pm 0.07 .
$$


With this value of $m_{0}$, the parameters $\widehat{g}_{1}$ and $\widehat{g}_{2}$ are: $\widehat{g}_{1}=10.2 \pm 0.7, \widehat{g}_{2}=17.3 \pm 0.8$. Notice that the performed fit is similar but not equal to the one of Ref. [8]: the inclusion of point (v) was not present in the fit of that work. As a consequence, the present values are slightly different from the ones of Ref. [8, but it turns out that the overall phenomenology does not change much (for instance, $m_{0}$ was determined to be $460 \pm 136 \mathrm{MeV}$ ). The biggest change is the increased (and less uncertain) value of the parameter $Z$, which is now in good agreement with the tree-flavor determination of Ref. [18. Having determined the parameters, it is possible to calculate the isospin-odd scattering length:

$$
a_{0}^{(-)}=(6.41 \pm 0.17) \cdot 10^{-4} \mathrm{MeV}^{-1}
$$

which is in very well agreement with the experimental value $a_{0, \exp }^{(-)}=(6.4 \pm 0.1) \cdot 10^{-4}$ measured at the Paul Scherrer Institute (PSI, Switzerland) with pionic hydrogen and deuterium X-ray experiments [29]. It is also interesting to compare our result with the one presented in Ref. [16, where the calculation of pion-nucleon scattering lengths was performed by using chiral perturbation theory (ChPT) including isospin-violating corrections: $a_{0, C h P T}^{(-)}=(6.16 \pm 0.06) \cdot 10^{-4} \mathrm{MeV}^{-1}$. Thus, our value is compatible with the ChPT result as well. Note, in our previous evaluation of Ref. 8 the scattering length had the value $a_{0}^{(-)}=(6.04 \pm 0.63) \cdot 10^{-4} \mathrm{MeV}^{-1}$, which is compatible with the result of Eq. (19). Interestingly, the new result has a smaller error in virtue of the improved fit.

The isospin-even scattering length $a_{0}^{(+)}$depends on the mass of the scalar-isoscalar $\sigma$. The $\sigma$-meson cannot be assigned unambiguously to a specific scalar listed in [1, see Refs. [17, 18, 30, 31] and refs. therein. Therefore, the isoscalar scattering length is plotted in Fig. 2 (left panel) for values of $m_{\sigma}$ varying between 400 and $1300 \mathrm{MeV}$. An agreement with the experimental (or even ChPT) band is possible for values of $m_{\sigma}$ smaller than $500 \mathrm{MeV}$. While such a low mass is not favoured by recent studies on light scalar states (the assignment $\sigma \equiv f_{0}(1370)$ is the favored one), the result shows that a low-scalar field, identified by the resonance $f_{0}(500)$ and interpreted as a tetraquark or a molecular state [30, 31, is needed. Although not present here, it can be easily coupled to the model, see Ref. [8, 10] and the discussions in Sec. 4.

A clear problem of the scenario with $N^{*}(1535)$ as the chiral partner is the theoretical result for the decay width $\Gamma_{N^{*} \rightarrow N \eta}^{\text {theor }}=4.9 \pm 0.8 \mathrm{MeV}$, which should be compared with the experimental value $\Gamma_{N^{*} \rightarrow N \eta}^{e x p}=63 \pm 10.5 \mathrm{MeV}$. Due to the mismatch in the decay channel $N^{*} \rightarrow N \eta$, one may wonder if the chiral partner of the nucleon is the resonance $N^{*} \equiv N(1650)$.

For the case $N^{*} \equiv N(1650)$ we follow the same strategy, but the numerical values for the axial coupling constant and the decay width refer now to the resonance $N(1650): g_{A}^{\left(N^{*}\right)}=0.55 \pm 0.2$ [26], $\Gamma_{N^{*} \rightarrow N \pi}^{e x p}=105 \pm 21 \mathrm{MeV}$ [1]. The parameters $m_{0}, c_{1}, c_{2}, \widehat{g}$, and $Z$ read $\left(\chi_{\min }^{2}=0.64\right)$

$$
m_{0}=659 \pm 146 \mathrm{MeV}, c_{1}=-2.80 \pm 0.21, c_{2}=13.2 \pm 0.8, Z=1.81 \pm 0.07 .
$$

The corresponding values of $\widehat{g}_{1}$ and $\widehat{g}_{2}$ are: $\widehat{g}_{1}=9.1 \pm 1.1, \widehat{g}_{2}=17.6 \pm 1.2$.

The result for the isospin-odd scattering length is similar to the previous scenario:

$$
a_{0}^{(-)}=(6.25 \pm 0.19) \cdot 10^{-4} \mathrm{MeV}^{-1}
$$

which is in well agreement with the experimental as well as the ChPT values quoted above. (Also in this case, the theoretical error is sizably smaller than in Ref. [8], where we had $a_{0}^{(-)}=(5.90 \pm 0.46) \cdot 10^{-4}$ $\left.\mathrm{MeV}^{-1}\right)$.

For the isospin-even scattering length a similar discussion holds, see Fig. 2 (right panel). Interestingly, there is no issue here with the decay into the $\eta$ meson: the theoretical decay width $\Gamma_{N^{*} \rightarrow N \eta}^{\text {ther }}=12.5 \pm 2.5$ $\mathrm{MeV}$ agrees well with the experimental value $\Gamma_{N^{*} \rightarrow N \eta}^{e x p}=15.0 \pm 3.0 \mathrm{MeV}$, thus rendering this scenario favoured.

We summarize the situation of the mirror assignment in Table 1 and Fig. 2; all the relevant experimental values are in Table 2 . 
As a last step, we turn to the discussion of the pion-nucleon sigma term, which in the present model with no explicit breaking term in the baryonic sector takes the form

$$
\sigma_{\pi N}=m_{N}-\left(m_{N}\right)_{\sigma_{0} \rightarrow \sigma_{0, \mathrm{CL}}},
$$

where $\sigma_{0, \mathrm{CL}}$ is the value of the chiral condensate in the chiral limit. Thus, $\sigma_{\pi N}$ represents the mass contribution which arises from the explicit symmetry breaking. Using the numerical values of Ref. [18] we obtain $\sigma_{0, \mathrm{CL}}=164 \mathrm{MeV}$, out of which

$$
\sigma_{\pi N} \simeq 15 \mathrm{MeV} .
$$

This value is slightly smaller than the recent lattice value $\sigma_{\pi N}=37 \pm 8 \pm 6[22$ ] and the theoretical evaluation based on the CHAOS data $\sigma_{\pi N}=44 \pm 12$ [23. A somewhat larger value $\sigma_{\pi N}=59 \pm 7$ $\mathrm{MeV}$ has been found by using chiral perturbation in Ref. 24]. It is then clear that our value, although smaller then what is found in other approaches, is non-negligible and shows that the explicit symmetry breaking encoded in the chiral condensate has an important effect on this quantity. The 'missing part' of $\sigma_{\pi N}$ could be easily accounted by including a standard mass-term for the nucleon fields. This step will also be crucial (together with the proper inclusion of all light scalar fields, see conclusions) for a more accurate determination of the isospin-even scattering length $a_{0}^{(+)}$. In fact, chiral perturbation theory shows that the quantities $\sigma_{\pi N}$ and $g_{A}$ directly enter in the lowest order expression of $a_{0}^{(+)}$and that the corresponding terms have the opposite sign, see e.g. Ref. 32. This is not the case for the isospin-odd scattering length, which in lowest-order chiral perturbation theory depends only on the nucleon mass, the pion mass, and the pion decay constant: $a_{0, \mathrm{LO}-\mathrm{ChPT}}^{(-)}=\left(1+m_{\pi} / m_{N}\right) \frac{m_{\pi}}{8 \pi f_{\pi}^{2}} \simeq 5.69 \cdot 10^{-4}$ $\left[\mathrm{MeV}^{-1}\right]$. This value is however about $15 \%$ too small when compared to the experimental result. In the framework of ChPT the gap is filled by NLO corrections [32, while in our approach the presence of additional mesonic and baryonic resonances allows to obtain the correct result. (The correspondence between the two approaches can be formally obtained by integrating out heavier resonances in our eLSM. When the latter are infinitely heavy, one obtains ChPT in lowest order).

Table 1: Summary of the results in the mirror assignment

\begin{tabular}{|l|l|l|}
\hline Quantity & $N(1535)$ & $N(1650)$ \\
\hline$m_{0}[\mathrm{MeV}]$ & $459 \pm 117$ & $659 \pm 146$ \\
\hline$a_{0, \text { theor }}^{(-)}\left[\mathrm{MeV}^{-1}\right]$ & $(6.41 \pm 0.17) \cdot 10^{-4}$ & $(6.25 \pm 0.19) \cdot 10^{-4}$ \\
\hline$\Gamma_{N^{*} \text { ther }}^{\text {th }}[\mathrm{MeV}]$ & $4.9 \pm 0.8$ & $12.5 \pm 2.5$ \\
\hline
\end{tabular}

Table 2: Values from experiment, ChPT and Lattice

\begin{tabular}{|l|l|}
\hline$a_{0, e x p}^{(-)}\left[\mathrm{MeV}^{-1}\right]$ & $(6.4 \pm 0.1) \cdot 10^{-4}$ \\
\hline$a_{0, e x p}^{(+)}\left[\mathrm{MeV}^{-1}\right]$ & $(-8.85 \pm 7.1) \cdot 10^{-6}$ \\
\hline$\Gamma_{N(1535) \rightarrow N \pi}^{e x p}[\mathrm{MeV}]$ & $67.5 \pm 11.2$ \\
\hline$\Gamma_{N(1650) \rightarrow N \pi}^{e x p}[\mathrm{MeV}]$ & $105 \pm 21$ \\
\hline$\Gamma_{N(1535) \rightarrow N \eta}^{e x p}[\mathrm{MeV}]$ & $63.0 \pm 10.5$ \\
\hline$\Gamma_{N(1650) \rightarrow N \eta}^{e x p}[\mathrm{MeV}]$ & $15.0 \pm 3.0$ \\
\hline$a_{0, C h P T}^{(-)}\left(\right.$from [16]) $\left[\mathrm{MeV}^{-1}\right]$ & $(6.16 \pm 0.06) \cdot 10^{-4}$ \\
\hline$a_{0, C h P T}^{(+)}\left(\right.$from [16]) $\left[\mathrm{MeV}^{-1}\right]$ & $(5.44 \pm 0.22) \cdot 10^{-5}$ \\
\hline$g_{A}^{N(1535)}$ (from [26]) & $0.2 \pm 0.3$ \\
\hline$g_{A}^{N(1650)}$ (from [26]) & $0.55 \pm 0.2$ \\
\hline
\end{tabular}

In conclusion, the mirror assignment provides a good description of the phenomenology. The only open issue for the choice $N(1535)$ is the theoretically too small decay width $\Gamma_{N^{*} \rightarrow N \eta}$, while the choice 
$N(1650)$ is in good agreement with all the values. In the future, one could try to combine both scenarios by including also the positive-parity Roper state $N(1440)$ and studying a four-state mixing problem [8]. Another interesting possible source of an enhancement of the decay of $N(1535)$ into $N \eta$ is the inclusion of terms involving the chiral anomaly in the baryonic sector. Namely, our Lagrangian (5) respects the $U(1)_{A}$ symmetry (the breaking of it is confined to the mesonic sector). However, in order to limit the free parameters of the model, a detailed study of the effect of the anomaly in the baryonic sector can be undertaken only when baryons with strangeness are included and thus much more experimental constraints can be put on the model.

\subsection{Naive assignment}

For $N^{*} \equiv N(1535)$ as the chiral partner of the nucleon, we determine the four free parameters $\tilde{c}_{1}, \tilde{c}_{2}$, $\tilde{c}_{12}$, and $Z$ by performing the very same fit done in the mirror assignment, leading to the following results $\left(\chi_{\min }^{2}=0.64\right)$ :

$$
\tilde{c}_{1}=-2.3 \pm 0.1, \tilde{c}_{2}=7.1 \pm 2.7, \tilde{c}_{12}=-0.9 \pm 0.1, Z=1.81 \pm 0.07
$$

The parameters $\tilde{\widehat{g}}_{1}$ and $\tilde{\widehat{g}}_{2}$ are given by the masses of the nucleons and $\sigma_{0}=167 \mathrm{MeV}$ through eq. (15): $: \tilde{\widehat{g}}_{1}=5.6 \pm 0.6, \tilde{\widehat{g}}_{2}=9.2 \pm 1.0$.

The result for the isospin-odd scattering length is (see Appendix A for the analytical expression ):

$$
a_{0}^{(-)}=(4.32 \pm 0.34) \cdot 10^{-4} \mathrm{MeV}^{-1}
$$

This value is not in agreement with experimental result $a_{0, \text { exp }}^{(-)}=(6.4 \pm 0.1) \cdot 10^{-4} \mathrm{MeV}^{-1}$ [29]. Namely, it is off by several standard deviations from the experimental value. This mismatch is an evident drawback of the naive assignment. (Namely, in this case the influence of the heavier resonances does not improve, but worsens the results of the lowest-order ChPT.)

Also in the naive assignment, the isospin-even scattering length $a_{0}^{(+)}$depends on the mass of the scalarisoscalar $\sigma$, see Appendix B for its explicit form. The quantity $a_{0}^{(+)}$is plotted as function of $m_{\sigma}$ in Fig. 2, left panel. We observe that the value of $a_{0}^{(+)}$reaches the experimental band for $m_{\sigma}=1000$ $1300 \mathrm{MeV}$. At first sight this result seems even in better agreement with the experiment than the mirror assignment, because the favoured mass of $\sigma$ is approximately $1.3 \mathrm{GeV}$ [17. However, care is needed: As mentioned above, in the present approach the lightest scalar state $f_{0}(500)$ is not present (in either assignment), although the latter resonance is known to couple strongly to nucleons, see also the discussions in Refs. [10, 33] and in the conclusions. Therefore, at the present stage an agreement with data is actually misleading.

We now turn to the decay $N^{*} \rightarrow N \eta$, for which we obtain the result

$$
\Gamma_{N^{*} \rightarrow N \eta}=4.9 \pm 0.8 \mathrm{MeV}
$$

which is considerably smaller than the experimental value $\Gamma_{N^{*} \rightarrow N \eta}^{\exp }=63.0 \pm 10.5 \mathrm{MeV}$ [1].

Table 3: Summary of the results in the naive assignment

\begin{tabular}{|l|l|l|}
\hline Quantity & $N(1535)$ & $N(1650)$ \\
\hline$a_{0, \text { theor }}^{(-)}\left[\mathrm{MeV}^{-1}\right]$ & $(4.32 \pm 0.34) \cdot 10^{-4}$ & $(4.31 \pm 0.34) \cdot 10^{-4}$ \\
\hline$\Gamma_{N^{*} \rightarrow N \eta}[\mathrm{MeV}]$ & $4.98 \pm 0.8$ & $12.5 \pm 2.5$ \\
\hline
\end{tabular}

If we choose $N^{*} \equiv N(1650)$ as the parity partner of the nucleon in the naive assignment, we get very small modifications of the scattering lengths (see Table 2 for the isospin-odd one, Fig. 2 (right panel) for the isospin-even one), but the decay width $\Gamma_{N^{*} \rightarrow N \eta}^{\text {exp }}=15 \pm 3 \mathrm{MeV}$ agrees with the experimental value just as in the mirror assignment. We summarize the situation of the naive assignment in Table 3 and Fig. 2. Note, in Fig. 2 only one curve for $a_{0}^{+}=a_{0}^{+}\left(m_{\sigma}\right)$ is shown in the naive assignment: this is 


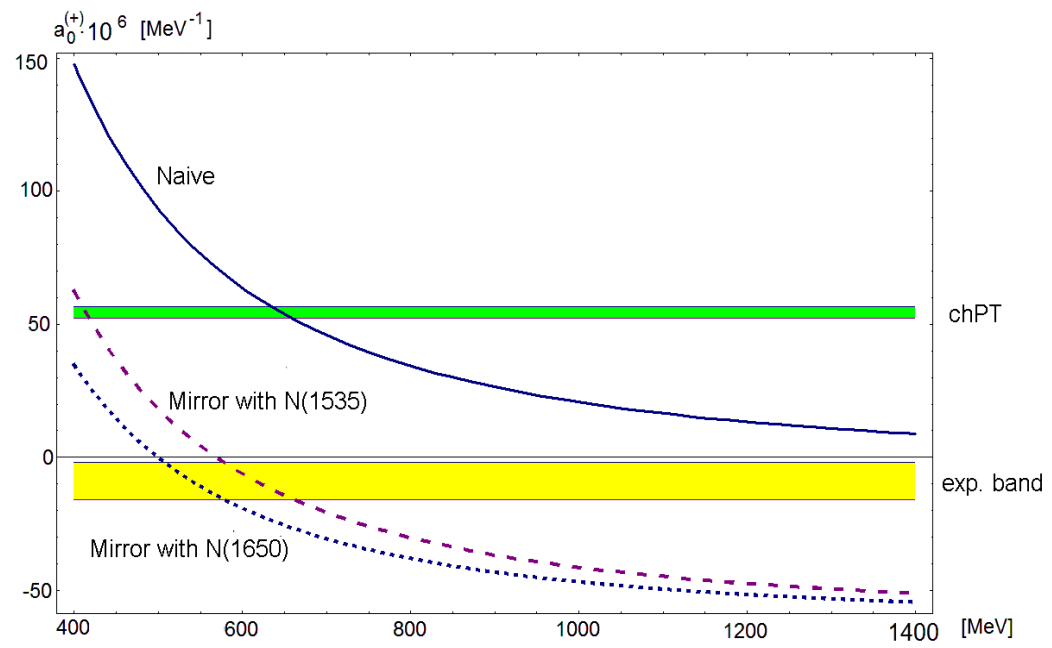

Figure 2: The isoscalar scattering length $a_{0}^{(+)}$is plotted as a function of $m_{\sigma}$ in the mirror assignment (dashed curve for the partner $N(1535)$, dotted curve for the partner $N(1650)$ ) and in the naive assignment (solid line, which is valid for both partners $N(1535)$ and $N(1650)$ ). The experimental range is shown by the lower band. The chPT result of Ref. [16] is represented by the upper narrow band.

due to the fact that the results for $a_{0}^{+}$are almost identical for the two partners $N(1535)$ and $N(1650)$. The small numerical difference cannot be resolved graphically.

A last comment concerns the pion-nucleon sigma term in the naive assignment: it amounts to $\sigma_{\pi N} \simeq 18$ $\mathrm{MeV}$, thus only slightly larger than in the mirror case. A similar discussion to the one in Sec. 3.1 holds here.

\section{Discussions and conclusions}

In this paper we have investigated the nucleon and its chiral partner, identified with either $N(1535)$ or $N(1650)$, within the extended Linear Sigma Model [8, 17, 18. The novel ingredient is that the chiral partner was incorporated in the naive assignment in presence of (axial-)vector degrees of freedom. The results were compared to the ones obtained in [8, where the chiral partner was coupled in the mirror assignment.

Two fundamental differences between the two assignments have played an important role in this work:

- In the naive assignment, the mass generation takes place only through the spontaneous breakdown of chiral symmetry. On the contrary, in the mirror assignment the mass term

$$
\mathcal{L}_{m_{0}}=-m_{0}\left(\bar{\Psi}_{2} \gamma_{5} \Psi_{1}-\bar{\Psi}_{1} \gamma_{5} \Psi_{2}\right)
$$

is allowed, and the mass of the nucleon is generated by this term and by the mechanism of chiral symmetry breaking. The quantity $\mathcal{L}_{m_{0}}$ is the only term in the baryonic sector of the eLSM Lagrangian which does not fulfill dilatation invariance. In order to achieve this in a chirally invariant way, which is one of the basic principle toward the construction of the eLSM [17, 18, one can modify $\mathcal{L}_{m_{0}}$ by including an interaction of the nucleon with a dilaton/glueball field $G$ and a tetraquark field $\chi$ (see Refs. [30, 31] and refs. therein for a phenomenological discussion):

$$
\mathcal{L}_{m_{0}} \rightarrow \mathcal{L}_{\chi G}=-(a \chi+b G)\left(\bar{\Psi}_{2} \gamma_{5} \Psi_{1}-\bar{\Psi}_{1} \gamma_{5} \Psi_{2}\right) .
$$


The mass term $m_{0}$ is then generated when shifting these fields by their vacuum expectation values, $\chi \rightarrow \chi_{0}$ and $G \rightarrow G_{0}$ :

$$
m_{0}=a \chi_{0}+b G_{0} .
$$

This fact shows an important point: even if the scalar-isoscalar field $\sigma$ (i.e., the chiral partner of the pion) is identified with the heavy resonance $f_{0}(1370)$, as current results show [17, 18, 30, 31, in the mirror assignment it is possible to write down a chirally invariant interaction which couples the nucleon to the lightest scalar-isoscalar light meson $f_{0}(500)$, which can be identified with a tetraquark field $\chi$ (or, similarly, to a pion-pion molecular state [30, 31]: the difference between these two configurations is not important here). Moreover, one can couple the nucleon also to the glueball field (to be identified predominantly with $f_{0}(1500)$ or with $f_{0}(1710)$ ). These additional interactions are not possible in the naive assignment without breaking chiral symmetry. This is a clear theoretical advantage of the mirror assignment w.r.t. the naive assignment. These properties are also important for the study of the model at non-zero density: in Ref. [10] it has been shown that a light tetraquark field $\chi \equiv f_{0}(500)$ allows for a correct description of nuclear matter saturation and compressibility.

- In the naive assignment the interaction between $N$ and $N^{*}$ is solely given by the derivative coupling to the pion, which in turn is possible only due to the inclusion of the (axial-)vector mesons in the model. Without (axial-)vector mesons the decay $N^{*} \rightarrow N \pi$ or the formation of a resonance $N^{*}$ in $\pi N$ scattering does not take place. In the mirror assignment we do not have such restrictions. Even without (axial-)vector mesons the decay $N^{*} \rightarrow N \pi$ does not vanish since terms of the form $\bar{N} i \gamma_{5} \vec{\pi} \cdot \vec{\tau} N^{*}$ are present.

In the mirror assignment, the theoretical value of the isospin-odd scattering length $a_{0}^{(-)}$is in very well agreement with experimentally measured and the ChPT results (see Tables 1 and 2). This is not the case in the naive assignment (Table 3), in which the theoretical result $a_{0}^{(-)}$is clearly too small. The isospin-even scattering is not yet conclusive (see Fig. 2): one has to go beyond the present treatment by including both resonances $f_{0}(500)$ and $f_{0}(1370)$ in the study of the scattering lengths, see also Refs. [10, 33. The decay into $N \eta$ turns out to bee too small for $N(1535)$ in any assignment, whereas for $N(1650)$ it agrees with the experimental data, thus favouring the latter as the chiral partner of the nucleon. Summarizing, our outcome is the following: the mirror assignment with the resonance $N(1650)$ as the chiral partner of the nucleon seems to be the favoured situation. Future studies on baryons are necessary to confirm that the mirror assignment is the correct way to incorporate the nucleon and its chiral partner. In the vacuum, an important future work is the description in a unique framework of the four baryonic resonances $N(940), N(1440), N(1535)$, and $N(1650) 8$. In this enlarged scenario it will be possible to test further the mirror and the naive assignments (and eventually a combination of them). Important hints come also from studies at nonzero density [10, 13, where it is shown that the mirror assignment is able to describe nuclear matter at nonzero density; the results do not depend on the mass of the chiral partner (and thus on the choice of $N(1535)$ or $N(1650)$ ), but depend strongly on the value of $m_{0}$. Namely, only for $m_{0} \gtrsim 500 \mathrm{MeV}$ the nuclear matter compressibility can be correctly described [10]. The range $m_{0} \gtrsim 500 \mathrm{MeV}$ is compatible with $N(1535)$, for which we have $m_{0}=459 \pm 117 \mathrm{MeV}$, but also (and even better) with $N(1650)$, for which $m_{0}=659 \pm 146 \mathrm{MeV}$. The need of a sizable $m_{0}$ was also corroborated in the recent study of Ref. 34, in which the emergence of an inhomogenous chiral condensate was studied: it was shown that a value of $m_{0} \gtrsim 500 \mathrm{MeV}$ is necessary to have an homogeneous liquid nuclear matter ground state.

In this paper we have also evaluated the pion-nucleon sigma term, which turns out to be about 15 $\mathrm{MeV}$ in the mirror case and about $18 \mathrm{MeV}$ in the naive one. Both values are smaller than the results present in the literature. This is due to the fact that in our approach no explicit symmetry breaking is considered in the baryonic part of the Lagrangian(s) and the pion-nucleon sigma terms arise solely from the explicit breaking present in the chiral condensate. The result represents anyhow a sizable contribution to the pion-nucleon sigma term. The missing piece can be included by adding an explicit 
breaking mass term for the baryons. This is an important outlook, which is also necessary for a better description of the isospin-even scattering length.

Considering that the calculation of scattering lengths and decays was carried at tree-level, an interesting outlook is the inclusion of mesonic loops. However, many resonances are present in the model, which are exchanged in scattering processes (as, for instance, the (axial-)vector mesons); their contribution is automatically taken into account at tree-level without the need of resummations. Moreover, the ratio of the decay width to the mass of the $N^{*}$ resonance (in both scenarios) is small, implying that the evaluation of loops, while surely valuable, is not expected to change the qualitative picture that we have presented 35 .

In future studies one should also incorporate the $\Delta$ resonance [36, 37 and extend the present model to $N_{f}=3$ also in the baryonic sector.

Acknowledgments: The authors thank D. H. Rischke, G. Pagliara, K. Teilab, C. Sasaki, M. Rho, A. Heinz, H. K. Lee, and J. Schaffner-Bielich for useful discussions. S. G. acknowledges support by the BMBF (Projekt 05P09RFFTF) and F. G. by the 'Stiftung Polytechnische Gesellschaft - Frankfurt am Main'.

\section{A Low-energy phenomenology}

In this appendix we present the formulae for the isospin-even and isospin-odd $s$-wave scattering lengths, for the axial coupling constant of the nucleon and its chiral partner, and for the decay widths $\Gamma_{N^{*} \rightarrow N \pi}$ and $\Gamma_{N^{*} \rightarrow N \eta}$. It should be stressed that the results presented here are based on a tree-level calculation. The isospin-even scattering length takes the form:

$$
\begin{aligned}
a_{0}^{(+)} & =\frac{1}{4 \pi\left(1+\frac{m_{\pi}}{m_{N}}\right)}\left\{-2 Z^{2} w^{2} \tilde{c}_{12}^{2}\left(m_{N^{*}}-m_{N}\right)\left[1+\frac{\left(m_{N^{*}}^{2}-m_{N}^{2}\right)\left(m_{N}^{2}+m_{\pi}^{2}-m_{N^{*}}^{2}\right)}{\left(m_{N}^{2}+m_{\pi}^{2}-m_{N^{*}}^{2}\right)^{2}-4 m_{N}^{2} m_{\pi}^{2}}\right]\right. \\
& -4 Z^{2} w \tilde{c}_{1}\left(\widetilde{\widehat{g}}_{1}-\tilde{c}_{1} w m_{N}\right)+\frac{\tilde{\tilde{g}}_{1}}{m_{\sigma}^{2}}\left[2 g_{1} w m_{\pi}^{2}+\frac{Z}{f_{\pi}}\left(m_{\sigma}^{2}-\frac{m_{\pi}^{2}}{Z^{2}}\right)-2 \frac{Z w^{2} m_{\pi}^{2} m_{1}^{2}}{f_{\pi}}\right] \\
& \left.+4 m_{\pi} m_{N} Z^{2}\left[w^{2} \tilde{c}_{12}^{2} \frac{m_{\pi}\left(m_{N^{*}}-m_{N}\right)^{2}}{\left(m_{N}^{2}+m_{\pi}^{2}-m_{N^{*}}^{2}\right)^{2}-4 m_{N}^{2} m_{\pi}^{2}}-\frac{\left(\widetilde{\widehat{g}}_{1}-2 \tilde{c}_{1} w m_{N}\right)^{2}}{4 m_{\pi}\left(m_{N}^{2}-m_{\pi}^{2}\right)}\right]\right\} .
\end{aligned}
$$

The isospin-odd scattering length is given by:

$$
\begin{aligned}
a_{0}^{(-)} & =\frac{1}{4 \pi\left(1+\frac{m_{\pi}}{m_{N}}\right)}\left\{4 Z^{2} w^{2} \tilde{c}_{12}^{2} \frac{m_{N} m_{\pi}\left(m_{N^{*}}-m_{N}\right)^{2}\left(m_{N^{*}}+m_{N}\right)}{\left(m_{N}^{2}+m_{\pi}^{2}-m_{N^{*}}^{2}\right)^{2}-4 m_{N}^{2} m_{\pi}^{2}}\right. \\
& -m_{\pi} 2 Z^{2} w^{2} \tilde{c}_{12}^{2}\left[1+\frac{\left(m_{N^{*}}-m_{N}\right)^{2}\left(m_{N}^{2}+m_{\pi}^{2}-m_{N^{*}}^{2}\right)}{\left(m_{N}^{2}+m_{\pi}^{2}-m_{N^{*}}^{2}\right)^{2}-4 m_{N}^{2} m_{\pi}^{2}}\right] \\
& \left.-2 Z^{2} \frac{\left(\tilde{\widehat{g}}_{1}-2 \tilde{c}_{1} w m_{N}\right)^{2}}{4 m_{N}^{2}-m_{\pi}^{2}}-2 Z^{2} w^{2} \tilde{c}_{1}^{2}+\frac{2 w \tilde{c}_{1}}{Z f_{\pi}}\left(\frac{m_{a}^{2}}{m_{\rho}^{2}}\right)\right\} .
\end{aligned}
$$

We have verified that the scattering lengths vanish in the chiral limit, $m_{\pi} \rightarrow 0$, as required by the low-energy theorems of QCD.

The expression for the decay width $N^{*} \rightarrow N \pi$ is:

$$
\Gamma_{N^{*} \rightarrow N \pi}=3 \frac{k_{\pi}}{2 \pi} \frac{m_{N}}{m_{N^{*}}} \frac{Z^{2} w^{2} \tilde{c}_{12}^{2}}{2}\left[\left(m_{N^{*}}^{2}-m_{N}^{2}-m_{\pi}^{2}\right) \frac{E_{\pi}}{m_{N}}+m_{\pi}^{2}\left(1-\frac{E_{N}}{m_{N}}\right)\right]
$$

where the momentum of the pseudoscalar particle is given by

$$
k_{\pi}=\frac{1}{2 m_{N^{*}}} \sqrt{\left(m_{N^{*}}^{2}-m_{N}^{2}-m_{\pi}^{2}\right)^{2}-4 m_{N}^{2} m_{\pi}^{2}}
$$


and where the energies are $E_{\pi}=\sqrt{k_{\pi}^{2}+m_{\pi}^{2}}$ and $E_{N}=\sqrt{k_{\pi}^{2}+m_{N}^{2}}$. Note, $\Gamma_{N^{*} \rightarrow N \pi}$ vanishes in the limit in which the (axial-)vector meson decouple, $g_{1}=0 \rightarrow w=0$.

Finally, the expression for the decay width $N^{*} \rightarrow N \eta$ takes the form

$$
\Gamma_{N^{*} \rightarrow N \eta}=\cos ^{2} \phi \frac{k_{\eta}}{2 \pi} \frac{m_{N}}{m_{N^{*}}} \frac{Z^{2} w^{2} \tilde{c}_{12}^{2}}{2}\left[\left(m_{N^{*}}^{2}-m_{N}^{2}-m_{\eta}^{2}\right) \frac{E_{\eta}}{m_{N}}+m_{\eta}^{2}\left(1-\frac{E_{N}}{m_{N}}\right)\right]
$$

where $k_{\eta}$ and $E_{\eta}$ are the momentum and the energy of the $\eta$-meson. The factor $\cos ^{2} \phi$ comes from the fact that the flavour wave function of the $\eta$-meson is given by

$$
\eta=\eta_{N} \cos \phi+\eta_{S} \sin \phi
$$

with $\eta_{N} \equiv(\bar{u} u+\bar{d} d) / \sqrt{2}$ and $\eta_{S} \equiv \bar{s} s$. Hence, the decay amplitude $\mathcal{A}_{N^{*} \rightarrow N \eta}$ should be written as:

$$
\mathcal{A}_{N^{*} \rightarrow N \eta}=\mathcal{A}_{N^{*} \rightarrow N \eta_{N}} \cos \phi+\mathcal{A}_{N^{*} \rightarrow N \eta_{S}} \sin \phi .
$$

We neglect $\mathcal{A}_{N^{*} \rightarrow N \eta_{S}}$ because it is OZI-suppressed and we use the mixing angle $\phi=-40^{\circ}$, in the middle of the range given in Eq. [38]

\section{B Naive model without (axial-)vector mesons: decoupling of $N$ and $N^{*}$}

The naive coupling constants of Eq. (12) are linked to those of Eq. (14) in the following way:

$$
\begin{aligned}
& \tilde{\widehat{g}}_{1}=\frac{-1}{4 \cosh \delta}\left(2 \widehat{g}_{12}+\widehat{g}_{1} e^{\delta}-\widehat{g}_{2} e^{-\delta}\right), \\
& \tilde{\widehat{g}}_{2}=\frac{-1}{4 \cosh \delta}\left(2 \widehat{g}_{12}-\widehat{g}_{1} e^{-\delta}+\widehat{g}_{2} e^{\delta}\right), \\
& \tilde{c}_{1}=\frac{1}{4 \cosh \delta}\left(2 c_{12}+c_{1} e^{\delta}+c_{2} e^{-\delta}\right), \\
& \tilde{c}_{2}=\frac{1}{4 \cosh \delta}\left(-2 c_{12}+c_{1} e^{-\delta}+c_{2} e^{\delta}\right), \\
& \tilde{c}_{12}=\frac{1}{4 \cosh \delta}\left(-2 c_{12} \sinh \delta+c_{1}-c_{2}\right) .
\end{aligned}
$$

As a last step we show explicitly the decoupling of the nucleon and the chiral partner in the naive assignment without (axial-)vector mesons. In this case the Lagrangian (12) takes the form:

$$
\begin{aligned}
\mathcal{L}_{\text {naive }} & =\bar{\Psi}_{1} i \gamma_{\mu} \partial^{\mu} \Psi_{1}-\widehat{g}_{1} \bar{\Psi}_{1}\left(\sigma+i \gamma_{5} \vec{\tau} \cdot \vec{\pi}\right) \Psi_{1}+\bar{\Psi}_{2} i \gamma_{\mu} \partial^{\mu} \Psi_{2}-\widehat{g}_{2} \bar{\Psi}_{2}\left(\sigma+i \gamma_{5} \vec{\tau} \cdot \vec{\pi}\right) \Psi_{2} \\
& -\widehat{g}_{12} \bar{\Psi}_{1}\left(\gamma_{5} \sigma+i \vec{\tau} \cdot \vec{\pi}\right) \Psi_{2}+\widehat{g}_{12} \bar{\Psi}_{2}\left(\gamma_{5} \sigma+i \vec{\tau} \cdot \vec{\pi}\right) \Psi_{1}+\ldots
\end{aligned}
$$

where we have set $c_{1}=c_{2}=c_{12}=g_{1}=w=0$ and where, for simplicity, the scalar mesons as $\eta$ and $\vec{a}_{0}$ are omitted. After introducing the physical fields $N$ and $N^{*}$ as in eq. (3) we get:

$$
\mathcal{L}=\bar{N} i \gamma_{\mu} \partial^{\mu} N-\tilde{\widehat{g}}_{1} \bar{N}(\sigma+i \vec{\tau} \cdot \vec{\pi}) N+\bar{N}^{*} i \gamma_{\mu} \partial^{\mu} N^{*}-\tilde{\widehat{g}}_{1} \bar{N}^{*}(\sigma+i \vec{\tau} \cdot \vec{\pi}) N^{*},
$$

Thus, one has a full decoupling of the fields $N$ and $N^{*}$ : there is no interaction among them [6] Only by introducing the (axial-)vector mesons the desired interactions arise.

\section{References}

[1] J. Beringer et al. (Particle Data Group), Phys. Rev. D86, 010001 (2012) 
[2] B. W. Lee, Chiral Dynamics (Gordon and Breach, New York, 1972).

[3] V. Koch, nucl-th/9512029

[4] V. Dmitrasinovic and F. Myhrer, Phys. Rev. C 61 (2000) 025205 hep-ph/9911320.

[5] C. E. Detar and T. Kunihiro, Phys. Rev. D 39 (1989) 2805.

[6] D. Jido, M. Oka, and A. Hosaka, Prog. Theor. Phys. 106 (2001) 873 hep-ph/0110005]. D. Jido, Y. Nemoto, M. Oka and A. Hosaka, Nucl. Phys. A 671 (2000) 471 hep-ph/9805306.

[7] A. Hosaka, D. Jido and M. Oka, Prog. Theor. Phys. Suppl. 149 (2003) 203 hep-ph/0305258.

[8] S. Gallas, F. Giacosa and D. H. Rischke, Phys. Rev. D 82 (2010) 014004 arXiv:0907.5084 [hep$\mathrm{ph}]$.

[9] S. Gallas, F. Giacosa and D. H. Rischke, Acta Physica Polonica B Proceedings Supplement, 2009. S. Gallas, F. Giacosa and D. H. Rischke, PoS CONFINEMENT 8 (2008) 089 arXiv:0901.4043 [hep-ph]]. S. Wilms, F. Giacosa and D. H. Rischke, Int. J. Mod. Phys. E 16 (2007) 2388 nucl-th/0702076 [NUCL-TH]].

[10] S. Gallas, F. Giacosa and G. Pagliara, Nucl. Phys. A 872 (2011) 13 arXiv:1105.5003 [hep-ph]]. A. Heinz, F. Giacosa and D. H. Rischke, arXiv:1312.3244 [nucl-th].

[11] W. -G. Paeng, H. K. Lee, M. Rho and C. Sasaki, Phys. Rev. D 85 (2012) 054022 arXiv:1109.5431 [hep-ph]]. W. -G. Paeng, H. K. Lee, M. Rho and C. Sasaki, Phys. Rev. D 85 (2012) 054022 arXiv:1109.5431 [hep-ph]]. W. -G. Paeng, H. K. Lee, M. Rho and C. Sasaki, arXiv:1303.2898 [nucl-th].

[12] C. Sasaki and I. Mishustin, Phys. Rev. C 82 (2010) 035204 arXiv:1005.4811 [hep-ph]].

[13] D. Zschiesche, L. Tolos, J. Schaffner-Bielich and R. D. Pisarski, Phys. Rev. C 75 (2007) 055202 nucl-th/0608044.

[14] L. Y. Glozman, Phys. Rept. 444 (2007) 1 hep-ph/0701081. T. D. Cohen and L. Y. Glozman, Int. J. Mod. Phys. A 17 (2002) 1327 [hep-ph/0201242]. L. Y. Glozman, Phys. Lett. B 539 (2002) 257 hep-ph/0205072.

[15] N. Fettes and U. -G. Meissner, Nucl. Phys. A 676 (2000) 311 hep-ph/0002162. N. Fettes and U. -G. Meissner, Nucl. Phys. A 693 (2001) 693 [hep-ph/0101030].

[16] V. Baru, C. Hanhart, M. Hoferichter, B. Kubis, A. Nogga and D. R. Phillips, Phys. Lett. B 694 (2011) 473 arXiv:1003.4444 [nucl-th]].

[17] D. Parganlija, F. Giacosa and D. H. Rischke, Phys. Rev. D 82 (2010) 054024 arXiv:1003.4934 [hep-ph]]. S. Janowski, D. Parganlija, F. Giacosa and D. H. Rischke, Phys. Rev. D 84 (2011) 054007 [arXiv:1103.3238 [hep-ph]].

[18] D. Parganlija, P. Kovacs, G. Wolf, F. Giacosa and D. H. Rischke, Phys. Rev. D 87 (2013) 014011 arXiv:1208.0585 [hep-ph]].

[19] A. Heinz, S. Struber, F. Giacosa and D. H. Rischke, Phys. Rev. D 79 (2009) 037502 arXiv:0805.1134 [hep-ph]].

[20] Y. Nemoto, D. Jido, M. Oka and A. Hosaka, Phys. Rev. D 57 (1998) 4124 hep-ph/9710445.

[21] V. Dmitrasinovic, A. Hosaka and K. Nagata, Int. J. Mod. Phys. E 19 (2010) 91 arXiv:0912.2396 [hep-ph]]. 
[22] G. S. Bali, P. C. Bruns, S. Collins, M. Deka, B. Glasle, M. Gockeler, L. Greil and T. R. Hemmert et al., Nucl. Phys. B 866 (2013) 1 [arXiv:1206.7034 [hep-lat]].

[23] J. Stahov, H. Clement and G. J. Wagner, Phys. Lett. B 726 (2013) 685 [arXiv:1211.1148[nucl-th]].

[24] J. M. Alarcon, J. Martin Camalich and J. A. Oller, Phys. Rev. D 85 (2012) 051503 arXiv:1110.3797 [hep-ph]].

[25] S. Gasiorowicz and D. A. Geffen, Rev. Mod. Phys. 41 (1969) 531.

[26] T. T. Takahashi and T. Kunihiro, Phys. Rev. D 78 (2008) 011503 [arXiv:0801.4707 [hep-lat]]. T. T. Takahashi and T. Kunihiro, eConf C070910 (2007) 297 [Mod. Phys. Lett. A 23 (2008) 2340] arXiv:0711.1961 [hep-lat]].

[27] D. M. Asner et al. [CLEO Collaboration], Phys. Rev. D 61 (2000) 012002 hep-ex/9902022].

[28] F. Divotgey, L. Olbrich and F. Giacosa, Eur. Phys. J. A 49 (2013) 135 [arXiv:1306.1193 [hep-ph]].

[29] D. Gotta, F. Amaro, D. F. Anagnostopoulos, S. Biri, D. S. Covita, H. Gorke, A. Gruber and M. Hennebach et al., Lect. Notes Phys. 745 (2008) 165.

[30] C. Amsler and F. E. Close, Phys. Rev. D 53 (1996) 295 arXiv:hep-ph/9507326. W. J. Lee and D. Weingarten, Phys. Rev. D 61, 014015 (2000). arXiv:hep-lat/9910008; F. E. Close and A. Kirk, Eur. Phys. J. C 21, 531 (2001). arXiv:hep-ph/0103173. F. Giacosa, T. Gutsche, V. E. Lyubovitskij and A. Faessler, Phys. Rev. D 72, 094006 (2005). arXiv:hep-ph/0509247. F. Giacosa, T. Gutsche and A. Faessler, Phys. Rev. C 71, 025202 (2005) arXiv:hep-ph/0408085. H. Y. Cheng, C. K. Chua and K. F. Liu, Phys. Rev. D 74 (2006) 094005 arXiv:hep-ph/0607206. V. Mathieu, N. Kochelev and V. Vento, Int. J. Mod. Phys. E 18 (2009) 1 [arXiv:0810.4453 [hep$\mathrm{ph}]]$.

[31] R. L. Jaffe, Phys. Rev. D 15 (1977) 267. R. L. Jaffe, Phys. Rev. D 15 (1977) 281. R. L. Jaffe, Phys. Rept. 409 (2005) 1 [Nucl. Phys. Proc. Suppl. 142 (2005) 343] arXiv:hep-ph/0409065. L. Maiani, F. Piccinini, A. D. Polosa and V. Riquer, Phys. Rev. Lett. 93 (2004) 212002 arXiv:hep-ph/0407017. F. Giacosa, Phys. Rev. D 74 (2006) 014028 arXiv:hep-ph/0605191. A. H. Fariborz, R. Jora and J. Schechter, Phys. Rev. D 72 (2005) 034001 arXiv:hep-ph/0506170. A. H. Fariborz, Int. J. Mod. Phys. A 19 (2004) 2095. arXiv:hep-ph/0302133. M. Napsuciale and S. Rodriguez, Phys. Rev. D 70 (2004) 094043. F. Giacosa, Phys. Rev. D 75 (2007) 054007 arXiv:hep-ph/0611388.

[32] A. W. Thomas and W. Weise, The Structure of the Nucleon, Berlin, Germany: Wiley-VCH (2001) $389 \mathrm{p}$.

[33] R. Machleidt, Phys. Rev. C 63 (2001) 024001 arXiv:nucl-th/0006014.

[34] A. Heinz, F. Giacosa and D. H. Rischke, arXiv:1312.3244 [nucl-th].

[35] F. Giacosa and G. Pagliara, Phys. Rev. C 76 (2007) 065204 arXiv:0707.3594 [hep-ph]]. N. N. Achasov and A. V. Kiselev, Phys. Rev. D 70 (2004) 111901 arXiv:hep-ph/0405128.

[36] G. W. Carter, P. J. Ellis and S. Rudaz, Nucl. Phys. A 603 (1996) 367 [Erratum-ibid. A 608 (1996) 514] nucl-th/9512033..

[37] D. Jido, T. Hatsuda and T. Kunihiro, Phys. Rev. Lett. 84 (2000) 3252 hep-ph/9910375.

[38] E. P. Venugopal and B. R. Holstein, Phys. Rev. D 57, 4397 (1998) hep-ph/9710382. 